\title{
Repetition Blindness and Retrieval-Time Effects of Full- vs. Partial-Report Following the Rapid Serial Visual Presentation (RSVP) of Letters in Words
}

\author{
John R. Vokey and Scott W. Allen \\ University of Lethbridge
}

\begin{abstract}
Although commonly accepted as an encoding/representational/perceptual phenomenon, repetition deficits ("repetition blindness") in Rapid Serial Visual Presentation (RSVP) can be shown to be markedly influenced by retrieval-time tasks independently of item encoding. We demonstrate such influences in a series of within-participant experiments where retrieval conditions are unpredictably varied after items have been experienced. Repetition deficits are demonstrated when full report of the presented item is required and in partial-report conditions where the repeated letter is included in the retrieval cue but not in partial-report conditions where the repeated letter is not included in the retrieval cue. Such effects are not expected if repetition deficits in RSVP are thought to be principally a function of the encoding/representation/perception of the trial experience.
\end{abstract}

Keywords: RSVP, repetition blindness, repetition deficits, retrieval-time, type-token, full vs. partial report

Repeating a letter in the rapid serial visual presentation (RSVP) of a word often results in participants failing to recall one of the presentations, despite the rate of presentation being sufficient for the accurate recall of the other letters in the word, and in particular the accurate recall of similarly positioned letters in words lacking the repetition (Kanwisher, 1991; Kanwisher \& Potter, 1990; Park \& Kanwisher, 1994). Kanwisher (1987) labelled this phenomenon "repetition blindness", characterizing it as a failure to perceive and subsequently to report the second of the two occurrences of the repeated letter. This construct of a perceptual blindness as a function of the RSVP of non-contiguous repetitions has been generalized by Kanwisher (see, e.g., Kanwisher, Kim, \& Wickens, 1996; Epstein \& Kanwisher, 1999) to include the RSVP of repeated words in both lists and sentence frames (e.g., Kanwisher, 1987), the RSVP of repetitions of coloured letters and colour patches (Kanwisher, 1991), and even the successive, but positionallydisplaced, and also the simultaneous, but very rapid presentation of repeated letters within words and pseudo-words (e.g., Kanwisher, 1991).

This work was supported in part by a Natural Sciences and Engineering Research Council of Canada (NSERC) Discovery grant to JRV, and was approved by the Human Subjects Research Committee of the University of Lethbridge. Both authors are currently members of the Department of Psychology at the University of Lethbridge, Lethbridge, Alberta, Canada TIK 3M4, and may be contacted via email at vokey@ulelth.ca or allens@uleth.ca. The authors declare no conflicts of interest.

\section{The Type/Token Account}

To account for these repetition-based deficits, Kanwisher suggested that when the elements of an item, such as the letters in a word or the words in a sentence, are presented sufficiently rapidly, repetitions of the elements are perceived or encoded as an enhancement or strengthening of a pre-existing type, rather than registering as yet another token occurrence of a previously experienced element within the item. According to this view, although participants may arrive at the subsequent report or recall task with evidence that $a$ token of the repeated element occurred in the RSVP of the item, they have no independent evidence in the form of a remembered perception that more than one exemplar of that element was experienced. For example, in the canonical description of Kanwisher (1991), although participants may be particularly likely to recall a P following the RSVP of the word POPLAR, ${ }^{1}$ possibly even to a greater extent than following the RSVP of the control word MAPLES, they will be less likely to recall that there were two Ps in POPLAR than that there were both an $M$ and a $P$ in MAPLES, preferring instead to report the RSVP of POPLAR as POLAR much more frequently than they report the RSVP of MAPLES as MALES. According to the type/token account, then, participants tend to fail to report the second of the two RSVP presentations of a repetition within an item because they have no independent perceptual evidence (beyond the

\footnotetext{
${ }^{1}$ Throughout this article, experimental stimuli are presented in a monospaced, typewriter font, much as they would occur on a computer screen in an experiment, to distinguish them from the expository prose.
} 
possible strengthening of the type) that it occurred, hence, the term "repetition blindness".

There are two critical features of the type/token account that should be emphasised (cf. Whittlesea, Dorken, \& Podrouzek, 1995; Whittlesea \& Podrouzek, 1995; Whittlesea $\&$ Wai, 1997). The first (and a feature common to all accounts that locate the repetition deficit in perception) is that the participants' recall or report of their RSVP experiences is veridical: differences in report are to be equated with differences in perception. If repetitions are reported less frequently following RSVP than are non-repetitions then it is because they are perceived or detected less frequently.

The second feature is that the type/token account (and its related variants, e.g., Hochhaus \& Marohn, 1991; Humphreys, Besner, \& Quinlan, 1988) implies two forms of memory, one for concepts or "types", and one for episodes or particular experienced exemplars ("tokens") of those types, that operate on different principles. Kanwisher et al. (1996), for example, go so far as to cite neuroanatomical evidence (Baylis, Driver, \& Rafal, 1993) for distinct "what" and "where" pathways in the visual system as what they refer to as a "functional correlate" of the relevant distinction between the two memory systems. Thus, following RSVP of a word containing repetitions, the "type" memory for the repeated letter is adequately activated and, hence, a letter of that type recognised as having occurred in the input stream, but, because of the RSVP, the episodic memory system is not able to function fully, and a distinct representation or record of the second occurrence of the letter is not created.

\section{The Retrieval-Time Processes Account}

Although Kanwisher prefers to locate the phenomenon in the perception of the item, accepting the participants' recall as a more or less unbiased, if possibly somewhat generally attenuated, report of their RSVP perceptual experience, it is also possible that the difficulty with the report of repetitions in a stream of rapid serially visually presented letters in words occurs not because the repetitions are less likely to be perceived (or encoded) as separate tokens or episodes, but because they often fail to be correctly (re)constructed and hence reported as such. That is, the difficulty may be directly with the retrieval-time report of repetitions within the RSVP of items, not necessarily the perception or encoding of them; in the normal course of recall it may simply be more difficult to remember or to construct for report repetitions qua repetition than it is to remember or to report upon unique, individual events, particularly when resources are sufficiently taxed at encoding, as in the RSVP of letters within words, such that our normal reconstruction or report strategies and mechanisms are forced to rely on at best partial or degraded and possibly ambiguous information. Under these conditions, the usually occult weaknesses and biases of these retrievaltime processes may be revealed, as may be their strategic and heuristical nature (cf. Armstrong \& Mewhort, 1995; Fagot \& Pashler, 1995; Greene, 1991; Masson, 2004; Masson, Caldwell, \& Whittlesea, 2000; Vokey \& Allen, 2002; Whittlesea \& Masson, 2005; Whittlesea et al., 1995; Whittlesea \& Podrouzek, 1995; Whittlesea \& Wai, 1997). ${ }^{2}$

In many respects, failing to perceive a repetition of a letter within the RSVP of a word and failing to recall or to report it immediately after it is presented would appear to amount to the same thing. Indeed, at the short exposure durations typically used in these tasks, one might argue that the distinction between perception and report is without functional merit. Yet, there are important differences from an analytic perspective. Chief among these is the difference in the kinds of tasks one would focus on in attempts to extend and to understand the phenomenon. Characterisations of the phenomenon as a perceptual failure have led Kanwisher and others to explore a broad range of perceptual and encoding conditions, providing for both useful extensions of and some limitations on the original findings, as in the series of experiments of Park and Kanwisher (1994). Characterising it as a retrieval-time phenomenon, on the other hand, suggests that investigating the conditions of retrieval or report of repetitions independently of the perceptual conditions of them may prove equally fruitful. Indeed, to the extent that the phenomenon is productively seen as a retrieval-time problem, there may little advantage to seeing it exclusively as a form of perceptual failure, or even a problem of perception at all (eg., Armstrong \& Mewhort, 1995; Fagot \& Pashler, 1995; Greene, 1991; Masson, 2004; Masson et al., 2000; Vokey \& Allen, 2002; Whittlesea \& Masson, 2005; Whittlesea et al., 1995; Whittlesea \& Podrouzek, 1995; Whittlesea \& Wai, 1997).

We present a series of experiments designed to gain control of the repetition deficit phenomenon through various manipulations of the conditions of retrieval to allow the participant to respond to repetitions. Although Kanwisher and her colleagues prefer to see the phenomenon as ubiquitous and presumably unitary in process, occurring with a wide variety of kinds stimuli and tasks (e.g., the RSVP of letters within words, words within sentence frames, colour patches within grids, etc.), we remain sceptical—not so much because we

\footnotetext{
${ }^{2}$ There are yet other accounts that do not clearly fall in one camp or the other. For example, Harris and her colleagues (e.g., Harris \& Morris, 2000; Harris, 2001) have proposed a pattern completion account of some of these repetition deficits that invokes sublexical recruitment of words containing the sublexical elements. Although the account is presented as principally perceptual with the subsequent report as an unbiased index of that perception, it equally could occur at retrieval. For example, in Experiment 2 of Harris (2001), the magnitude of the repetition deficit was found to covary with the number of words lexically available that were consistent with the non-repeated letters of the test items - an effect that could occur either at encoding as the perceptual representation is obtained or at retrieval as the recall is constructed in response to the evidence provided by the degraded RSVP input.
} 
are not convinced that a similar phenomenon can be made to occur with many different kinds of stimuli and testing conditions, but because we suspect that there are many and varied retrieval-time processes, some varying with changes in stimuli and testing conditions, that can be shown to be responsible, in part if not in full, for the difficulty with repetitions under the appropriate retrieval and report conditions. Thus, for this series of experiments, we investigate only the repetition deficit following RSVP of letters within word-strings as in the original Kanwisher (1991); Kanwisher and Potter (1990); Park and Kanwisher (1994) demonstrations (see also Armstrong $\&$ Mewhort, 1995). Whether the retrieval-time effects we observe with this constrained stimulus construct have greater generality over other encoding tasks, stimuli, and test tasks, such as to words within sentence frames, remains to be seen; we suspect that at some sufficiently abstract level of description, some of them do, but we believe it unwise to assume so a priori. The closely-related work of Whittlesea and Masson and their colleagues (Masson, 2004; Whittlesea \& Masson, 2005; Whittlesea et al., 1995; Whittlesea \& Podrouzek, 1995) on the repetition deficit of the RSVP of words in lists of words, though, suggests that the retrieval-time analysis of these phenomena may be more generally successful.

The initial Kanwisher (Kanwisher, 1991; Kanwisher \& Potter, 1990; Park \& Kanwisher, 1994) experiments and theorisation have spawned a large literature of subsequent research on repetition blindness, mostly focussing on the encoding/representational/perceptual approaches to the phenomenon. Although no doubt valuable and important in its own right, the majority of that work is irrelevant to the approach we take here concerning strictly the conditions of retrieval following the singular encoding during RSVP of a control vs. a repetition word. But, we would be remiss if we did not at least acknowledge some of the cleverness of much of this work. We found ourselves drawn, for example, to the work of Goldfarb and Treisman (2011) in which not only is presented an excellent and relatively recent summary of the encoding/representational/perceptual approaches to the phenomenon of RB, but an intriguing and more broadly generalisable perceptual approach involving the power of grouping.

\section{Overview of the Experiments}

The purpose of these experiments was to replicate and to extend the basic phenomenon in an experiment conducted by Kanwisher (1991) into the realm of retrieval-time phenomena. In the Kanwisher (1991) experiment, participants' reports of the RSVP of the letters of each of a dozen words containing a single, non-adjacent letter repetition (e.g., POPLAR) were compared with that of their matched control words lacking such a repetition (e.g., MAPLES). Matched in this case meant that a matched-pair of repetition and control items were of the same length, generally similar in meaning, had the same critical letter at the second or $\mathrm{P} 2$ position of the repeated letter in the repetition item, and that both words would produce a (different) English word (e.g., POLAR and MALES) if the critical letter in the P2 position were dropped, but neither would produce an English word if the letter in the first or $\mathrm{P} 1$ position of the repeated letter in the repetition item were dropped. Thus, ceteris paribus, participants should be no more likely to recall control words accurately than repetition words following the RSVP of the letters. However, Kanwisher (1991) found that participants were more accurate at recalling control words, principally because they were also more accurate at recalling the critical letters from the P1 and P2 positions in the control words than the repeated letter from both the P1 and P2 positions in the repetition words. That is, Kanwisher (1991) found that both whole-word recall (all letters correctly retrieved in order) and the recall of just the P1 and P2 letters (in any order) was superior for control items than for words containing nonadjacent repetitions.

Similar results have been reported for the recall of letters from the RSVP of pseudo-words and non-words (e.g., Kanwisher, 1991; Fagot \& Pashler, 1995), so it is clear that possible extra-experimental differences between repetition and non-repetition items are probably not necessary for the effect to occur; ${ }^{3}$ they may, however, be sufficient or at least contribute to the effect when real words are used. For example, if the control items of the pairs have a higher lexical frequency on average than their matched repetition items, then the effect might be expected to occur simply because the control words would be more readily available as plausible responses or reconstructions of the degraded record resulting from RSVP than would the repetition items. Similarly, it is possible that the words resulting from $\mathrm{P} 2$ deletions of repetition items but not (or at least less so of) those of control items are of higher lexical frequency on average than the test items themselves. It is also possible that the $\mathrm{P} 2$ deletion words from repetition items are, in general, of higher average lexical frequency than those of control items (e.g., POLAR from POPLAR may be of generally of higher lexical frequency than MALES from MAPLES, if only because of the shorter length, if nothing elsewe have no idea, we are just speculating). In either case, then, again, the repetition deficit might occur because these items would be more readily available as plausible, reconstructive responses than would their non-repetition control counterparts. Furthermore, the fact that the items in Kanwisher (1991) still produce words when the P2 letters are dropped, but not when the P1 letters are dropped also may have contributed to the effect, or at least to the conclusion that it is the second or repeated occurrence of the repeated letter that is disproportionately lost in these tasks (see Kanwisher, 1991; Whittlesea

\footnotetext{
${ }^{3}$ Even this conclusion is not strictly forced in that residual similarities of these pseudo- and non-word stimuli to real words or to the English orthography of pseudo-words or even to unpronounceable non-words (e.g., familiar consonant strings) may be sufficient to produce the effect.
} 
et al., 1995; Whittlesea \& Podrouzek, 1995, for a similar concern with the RSVP of words in sentence frames). There is also the concern that with such a small sample of 12 items the effect, or at least the bulk of it, may be peculiar to only a few of the control-repetition pairs, rather than being true in general of such pairs, despite the care Kanwisher (1991) took to ensure that the effects were statistically reliable across items (i.e., matched pairs) as well as participants. Relatedly, the effect obtained in this way may in fact be quite general, but only because the majority of control-repetition pairs formed in this way routinely result in one or more of these biases in the materials.

For these reasons, we collected a large set of words containing non-adjacent repetitions of a single letter. For each repetition word (e.g., STATE, PIPE) there was a same-length, non-repetition control word differing in spelling from the repetition word by only one letter in either the P1 (e.g., SLATE) or P2 (e.g., PILE) position of the repeated letter in the repetition item, and such that the item produced by dropping this letter was also an English word (e.g., SATE, PIE). The triplets thus formed were of two types. P1 triplets, such as STATE-SLATE-SATE, were composed of words that differed in the $\mathrm{P} 1$ position of the repetition in the repeated word; $\mathrm{P} 2$ triplets, such as PIPE-PILE-PIE, in contrast, were composed of words that differed in the $\mathrm{P} 2$ position of the repetition, as in the Kanwisher (1991) materials. In general, then, any of the three members of each triplet could be seen as a not unreasonable (i.e., fungible) response to the RSVP of either the same-length control or the repetition word. ${ }^{4}$

Common Materials. 140 triplets of English words were constructed. Each triplet consisted of one word containing a non-adjacent repetition of one of the letters (e.g., STATE, PIPE), a same-length, non-repetition control word in which one of the two repeating letters was replaced with a different letter to spell another English word (e.g., SLATE, PILE), and a deletion control word in which the letter that had been substituted to produce the same-length control was simply deleted to produce another English word one letter shorter in length (e.g., SATE, PIE). One-half of the triplets differed in the position of the first or P1 presentation of the repeated letter (e.g., STATE-SLATE-SATE), and the remaining triplets differed in the second or $\mathrm{P} 2$ presentation of the repeated letter (e.g., PIPE-PILE-PIE). None of the items of either triplet type produced legitimate words if the letter in the alternate repetition position were dropped. A small number of the triplets of each type (exact number depending on the experiment) were used to provide a fixed set (over participants in the experiment) of practise items during the initial trials of the testing phase, leaving over 100 critical triplets.

Common Procedure: Full vs. Partial Report. When the attributed meaning of report following the brief presentation of letters is ambiguous, one is immediately reminded of the classic work of Sperling (1960) regarding the recall of the tachistoscopic display of letter arrays. Sperling (1960) contrasted "full-report" of briefly-presented displays (analogous to the standard recall conditions of repetition blindness) with "partial-report" of those displays (signalled recall of just one line of the letter array) and showed that the report of a post hoc, randomly signalled, single line exceeded that predicted from full report (actually, the report was the same 3-4 letters in either case, but because the participants received 3 lines of 4 characters each, partial-report predicted, then, that participants must have had 9-12 letters available for report). The point was to demonstrate that there was much more available for report following brief displays than typically was captured with full report.

We adopt, in a fashion, that methodology here as our retrieval-time manipulation, contrasting within-participant full-report [i.e., the response following RSVP used in Kanwisher (1991)] with that of the partial report of a single post hoc flagged letter following standard repetition deficit RSVP conditions, just in case full report is a biased reading of the perceptual availability for report of RSVP displays of non-adjacent repetitions. It is important that the manipulation occur within-participant and following RSVP just in case participants would otherwise cope with the two tasks by changing the perceptual encoding of the RSVP of the items as a function of which response is requested. From standard perceptual/representation explanations of repetition blindness, then, one would presumably predict that the distinction here between full- vs. partial-report should be of no consequence for the repetition deficit: partial-report may be more accurate overall than full-report (à la Sperling, 1960), but the repetition deficit should, in general, be unchanged; that is, the full- vs. partial-report comparison should be unbiased with respect to the repetition deficit.

For each experiment, one-half of the critical items were tested with full-report: participants were cued following RSVP of the word to report as many letters as they could of the just presented item. That is, the instruction Please report the word followed by <return $>$ appeared on the screen along with an empty response box where they typed their response. The remaining critical items were tested with one or more partial-report conditions: participants were cued, following RSVP of the word, to report just one of the two critical letters at a flagged position of the just presented item (e.g., the L in PILE, the second P in PIPE, the

${ }^{4}$ Even with these additional manipulations and controls relative to the Kanwisher (1991) materials, however, the alternatives are not fully fungible. Retrieval of the non-repetition letter at the critical position (e.g., the L in PILE) when the same-length control item is presented in RSVP necessarily eliminates the remaining items of the triplet as fungible alternatives, whereas retrieving the repetition letter (in its correct, but non-critical position) when the repetition item is presented (e.g., the first P in PIPE) does not eliminate the remaining items of the triplet as plausible alternatives. 
$\mathrm{L}$ in SLATE, or the first T in STATE). Precisely how they were cued as to the position to report varied across experiments and are thus described separately for each experiment. Over participants, each triplet was rotated through the two fullvs. partial-report testing conditions, and participants were tested with the RSVP either of the same-length control word of a triplet (e.g., SLATE, PILE) or the repetition word (e.g., STATE, PIPE). The presentation order of items at test was independently randomised for each participant.

For each experiment, each participant was assigned to one of the four counterbalancing conditions that rotated the triplets through the two full- vs. partial-report $\times$ two samelength control vs. repetition test conditions, such that there were six participants in each counterbalancing condition, for a total of 24 participants in each experiment. All phases of the experiment were presented via computer, and each participant was tested at an individual computer work station.

In each experiment, participants were presented with all 140 of the test items, in upper-case, presented one at a time in the centre of the computer screen. Participants initiated each testing trial by pressing the space-bar on the computer keyboard. The first items, which were the same for every participant, were practise trials, and, unbeknownst to the participants, the data from these items were not used for analysis. Of the remaining items, one-half were same-length control items and the remainder were repetition items. For testing, each item received an ampersand as a prefix-mask and a percent-sign as a suffix-mask. These supplemented character strings were then displayed in what Kanwisher (1991) refers to as the "stationary RSVP" procedure. Each character of the string, except the last (the suffix-mask), was displayed in the centre of the computer screen for $133.33 \mathrm{~ms}(8 / 60 \mathrm{~s}-\mathrm{i} . e ., 8$ "ticks") before being over-written in the same position by the next character of the string (cf. Kanwisher, 1991). Following exposure for the same $133.33 \mathrm{~ms}$, the terminal mask character remained on the screen as the participant was then prompted for the full- or partial-report of the just-presented item. For partial report, the single-typed letter was recorded following the pressing of the return key to terminate the trial. For fullreport, participants were instructed to type as many letters of the presented word as they could recall, ignoring the initial and terminal mask characters, and they were free to edit each response until they were satisfied that they had responded as accurately as they could, which they indicated by pressing the return key on the keyboard to terminate the trial. The edited string was recorded by the computer, and served as the basis for the subsequent data analyses.

For full-report, a response was scored as correct if both of the critical letters from the P1 and P2 positions of the test word were included in the response, regardless of the reported position and any other letters that may have been reported. For partial-report, a response was scored as correct if the letter at the flagged position was reported correctly. The effect of interest, the repetition deficit, is evinced whenever the accurate recall associated with the control test condition reliably exceeds that of the corresponding repetition test condition.

\section{Experiment 1: Recall and Partial Report (All Letter Context)}

Full-report was as in Kanwisher (1991); Kanwisher and Potter (1990); Park and Kanwisher (1994). For partial report, we provided the full, all-letter context. For example, if a P1 item-set were used, such as SLATE-STATE, the test of partial-report would consist of the string ${ }_{\wedge}$ _ATE with a circumflex under the underscore to flag the recall of the letter at that position, and similarly if a P2 item-set were used, such as PIPE-PILE, except the circumflex would occur under the underscore ${ }^{\mathrm{PI}}{ }^{\mathrm{E}}$ replacing the second critical letter to flag the recall of the letter at that position (cf. Averbach \& Coriell, 1961; Averbach \& Sperling, 1961). From a retrievaltime perspective, the all-letter retrieval context we provide resembles at least to some degree the typical full-report recall context-recall letters in the context of other recalled lettersa potential source of recall bias that we revisit in detail in subsequent experiments. Participants were unaware of the required report task (full- or partial-report) until after the RSVP of the word for that trial.

\section{Method}

Participants. Twenty-four students of both sexes participated in exchange for partial credit in an introductory or second-year psychology course.

Materials and Design. The materials and design were the same as described in the overview section. Four items were used as practise items, leaving 136 items to be counterbalanced over conditions. One-half of the trials requested full-report, the remainder partial-report. Within each type of report, one-half of the trials were the RSVP of the samelength control item of the triplet, and the remainder were the RSVP of the repetition item for both P1 and P2 items.

\section{Results and Discussion}

As our principal concern is the repetition deficit, the analyses here were a series of simple, within-participant oneway ANOVAs of control vs. repetition items for full- vs. partial-report of P1 vs. P2 items-sets, along with the corresponding squared, point-biserial correlation coefficients $\left(r_{p b}^{2}\right)$ as a measure of the individual effect-sizes. Shown in Figure 1 are the mean proportion of correct responses to same-length control vs. repetition items, as a function of full- vs. partial-report, for each of the P1 (e.g., SLATE vs. STATE) and P2 (e.g., PILE vs. PIPE) item-sets. Errorbars are the unique Fisher $\mathrm{LSD}_{.05}$ for each control vs. repetition comparison. As may be seen, all four conditions 
VOKEY \& ALLEN

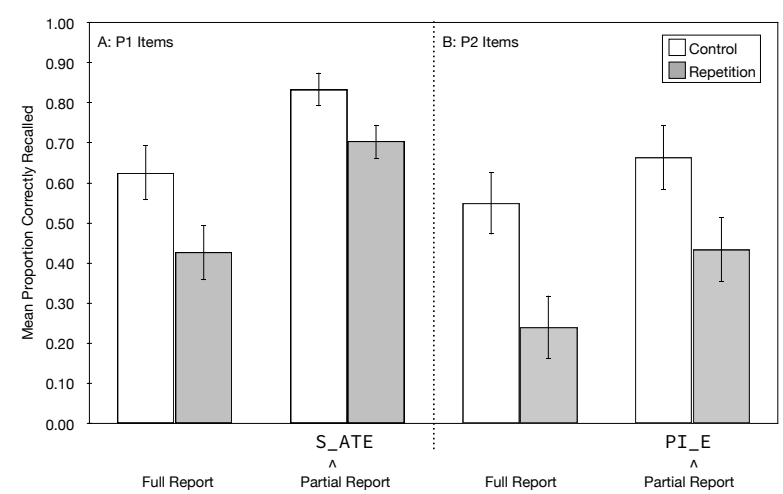

Figure 1. Mean proportion of correct recall in Experiment 1 as a function of repetition-type (control vs. repetition) for fulland partial-report for both P1 (panel A:) and P2 (panel B:) items. The cue for partial report included all letters except the to-be-reported letter. Error-bars are \pm Fisher $L S D_{.05}$ for the effect of repetition-type within each of the recall vs. partialreport conditions of the P1 vs. P2 item-sets.

evinced large repetition deficits: control words were correctly reported significantly more frequently than were repetition words in both full- and partial-report for both P1 $\left(F(1,23)=37.07, M S E=0.0128, p<.0001, r_{p b}^{2}=0.62\right.$ and $F(1,23)=40.81, M S E=0.0050, p<.0001, r_{p b}^{2}=0.64$, respectively $)$ and $\mathrm{P} 2(F(1,23)=66.5842, M S E=0.0172, p<$ $.0001, r_{p b}^{2}=0.74$ and $F(1,23)=34.45, M S E=0.0185, p<$ $.0001, r_{p b}^{2}=0.60$, respectively) item-sets.

Two conclusions follow: 1) it is possible to produce substantial repetition deficits with partial-report when a) whether partial-report would be required and $b$ ) which letter-position would be subject to report were not made available until after the RSVP of the word; and 2), more important, it is possible to maintain substantial repetition deficits with fullreport even in the context of unpredictable trials requiring partial-report. The latter result is maintained in all subsequent experiments, even as the repetition deficit is eliminated in subsequent partial report conditions. Thus, even if it were the case that requiring partial-report unpredictably on some trials resulted in participants shifting globally to a different form of perceptual encoding and representation on every trial (even though we have no reason to believe they would do so) than they would normally do so for strict full-report, that representation still results in repetition deficits in full-report, and, hence, provides no bases from the perceptual representation perspective to account for any such retrieval-time differences with partial-report.

\section{Experiment 2: Full- and Partial-Report (All and No Letter Contexts)}

Experiment 2 was a replication of Experiment 1, with one addition. As in Experiment 1, 50\% of the trials requested full-report; however, in Experiment 2, the remaining 50\% partial-report trials were split equally into two types: the allletters context of Experiment 1, and a no-letters context in which participants received a display of underscores equal in number to the number of letters of the word, with a circumflex under the underscore indicating the position of the letter to be recalled. The idea here was the possibility that the presence of the other letters in both full-report (as generated by the participant) and all-letter context partial report (as presented in the retrieval cue) interfered with the retrieval of the critical (repeated) letter on repetition trials, possibly because the occasional initial retrieval of the repeated letter in the fullreport condition and the presence of the repeated letter in the all-letter context condition provided a sufficient response to any recalled evidence that the repeated letter had occurred during RSVP: with that epistemic hunger at least sometimes satisfied, the participant may not feel compelled to report a second occurrence of the repeated letter. As in Experiment 1, participants were unaware of the required report task until after the RSVP of the word for that trial.

\section{Method}

Participants. Twenty-four students of both sexes participated in exchange for partial credit in an introductory or second-year psychology course.

Materials and Design. The materials and basic design were the same as described in Experiment 1, except that to counterbalance the items over the second partial report condition, 12 items ( $6 \mathrm{P} 1$ and $6 \mathrm{P} 2$ items) were used as practise items, leaving 128 items as the critical items. One-half of the trials requested full-report, the remainder partial-report, split here into two types of partial-report: all-letters (as in Experiment 1), and no-letters contexts. Within each type of report, one-half of the trials were the RSVP of the same-length control item of the triplet, and the remainder were the RSVP of the repetition item.

\section{Results and Discussion}

The data were analysed as in Experiment 1. Shown in Figure 2 are the mean proportion of correct responses to same-length control vs. repetition items, as a function of full-report, partial-report (all-letters context), and partialreport (no-letters context), for each of the P1 (e.g., SLATE vs. STATE) and P2 (e.g., PILE vs. PIPE) item-sets. Errorbars are the unique Fisher LSD.05 for each control vs. repetition comparison. As may be seen, all four of the conditions from Experiment 1 evinced large repetition deficits: control words were correctly reported significantly more 


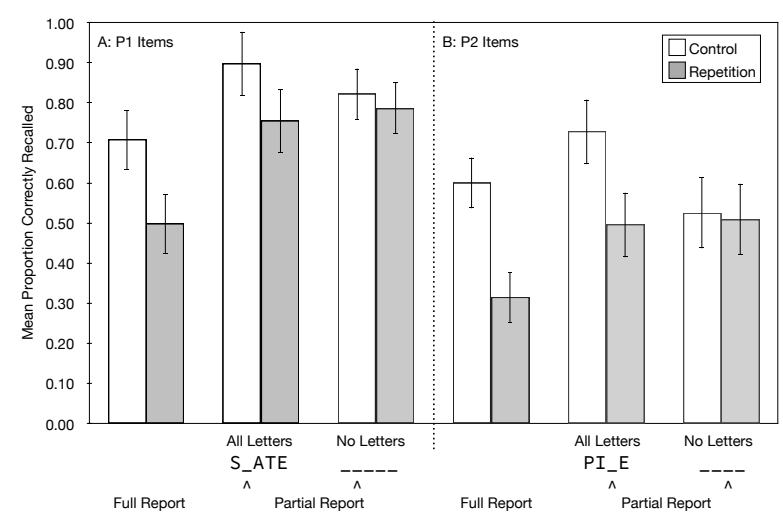

Figure 2. Mean proportion of correct recall in Experiment 2 as a function of repetition-type (control vs. repetition) for fulland partial-report (both all-letters vs. no-letters) for both P1 (panel A:) and P2 (panel B:) items. The cue for the all-letters condition included all letters except the to-be-reported letter whereas the cue for the no-letters condition replaced each letter with an underscore character. Error-bars are \pm Fisher $L S D_{.05}$ for the effect of repetition-type within each of the fullvs. partial-report (all-letters vs. no-letters) conditions of the $\mathrm{P} 1$ vs. P2 item-sets.

frequently than were repetition words in both full- and partial-report (all-letters context) for both $\mathrm{P} 1(F(1,23)=$ 34.02, $M S E=0.0181, p<.0001, r_{p b}^{2}=0.60$ and $F(1,23)=$ $13.45, M S E=0.0212, p<.0001, r_{p b}^{2}=0.37$, respectively) and $\mathrm{P} 2\left(F(1,23)=85.47, M S E=0.0134, p<.0001, r_{p b}^{2}=\right.$ 0.79 and $F(1,23)=34.57, M S E=0.0218, p<.0001, r_{p b}^{2}=$ 0.60 , respectively) item-sets.

However, that was not the case for partial-report in the no-letter context. As can be seen in Figure 2, there is no evidence of a repetition deficit for either P1 or P2 item-sets: control words were not correctly reported significantly more frequently than were repetition words $(F(1,23)=1.29, M S E=$ $0.0138, p=.2654, r_{p b}^{2}=0.05$ and $F(1,23)=0.38, M S E=$ $0.0262, p=.5414, r_{p b}^{2}=0.02$, for $\mathrm{P} 1$ and $\mathrm{P} 2$ items-sets, respectively). As the only difference between the all-letter and the no-letter partial-report contexts is the presence of the other letters of the word, it would appear to be the case that providing participants with the other letters (or have them provide them themselves, as in full-report) is at least one source of the repetition deficit. The result is ironic: as with the original repetition deficit (Kanwisher, 1991; Kanwisher \& Potter, 1990; Park \& Kanwisher, 1994) in which repetition at encoding, which is usually thought to be a benefit to performance, but instead appears to diminish it, providing a fullretrieval context relative to none at all, also seems to diminish rather than enhance retrieval of repetition items relative to same-length controls.

The contrast of full-report to the no-letter partial-report context is informative. Initially, following RSVP, they are very similar report conditions: neither has any other letters to cue retrieval, and they differ primarily in that one requires the report of as many letters as the participant can recall, and the other only the report of the letter at the one cued letter position. Yet, the former produces a substantial repetition deficit, and the latter little evidence for a repetition deficit at all. Inspection of Figure 2 suggests an explanation. Comparing the two means of the control conditions and the two means of the repetition conditions of full- and no-letter partial-report for each of the P1 and P2 data-sets, we find that the principle effect is that the no-letter partial-report condition increases retrieval of the repetition items relative to fullreport to much greater extent than, if at all, the control items for both P1 (control: $F(1,23)=6.20, M S E=0.0231, p=$ $.0204, r_{p b}^{2}=0.21$ and repetition: $F(1,23)=45.27, M S E=$ $\left.0.0202, p<.0001, r_{p b}^{2}=0.66\right)$ and P2 (control: $F(1,23)=$ $0.2447, M S E=0.0402, p=.6256, r_{p b}^{2}=0.01$ and repetition: $\left.F(1,23)=23.29, M S E=0.0224, p<.0001, r_{p b}^{2}=0.50\right)$ item-sets. That is, relative to no-letter partial-report, fullreport appears to interfere with the retrieval of repetition items, presumably as a consequence of the retrieval of other letters (including the repetition letter) in the word. ${ }^{5}$

\section{Experiment 3: Full- and Partial-Report (Critical Letter and Non-Critical Letter Contexts)}

Experiment 3 is a variant of Experiment 2. As in Experiments 1 and 2, 50\% of the trials required the standard, fullreport, and, as in Experiment 2, the remainder were divided equally between two partial-report contexts. As previously, both partial-report conditions required the recall of a single critical letter from a flagged position in the test word (e.g., the only or first T in SLATE or STATE, and the only or second P in PILE or PIPE). Each condition provided a retrieval context of

\footnotetext{
${ }^{5}$ There is one other difference and potential confound between the full-report and no-letter partial-report conditions: the latter through the list of underscore characters informs the participant of the number of letters in the word; perhaps that, in itself, provides an explanation for the difference between full- and no-letter partial-report and the presence or absence of a repetition deficit. Accordingly, we ran another experiment to control for this possibility (same materials, same number of participants, same counterbalancing, etc. as in Experiment 1). In this case, we compared the standard full-report with no indication as to the number of letters in the just presented word on $50 \%$ of the trials to another full-report condition, the "hint" condition, that used a display of underscores (as in the no-letter partial-report condition) to indicate the number of characters in the to-be-recalled word. The results were that the data for the two conditions were virtually identical, and both evinced significant and substantial repetition deficits for both P1 and P2 item-sets.
} 
a string of underscore characters indicating the length of the word with one letter of the word present as a retrieval cue. In the critical letter context, that letter was the alternate critical letter of the word (e.g., for P1 items: ${ }^{--}{ }^{-}{ }^{-}$for SLATE or STATE, and for P2 items: ${ }_{-}{ }_{-}-$for PILE or PIPE), with a circumflex under the underscore for the critical letter of that word indicating retrieval of the letter at that position. In the non-critical letter context, the retrieval context was the non-critical letter that flanked (either preceding or following, selected at random at runtime) the critical letter (e.g., for P1 items: ${ }_{\wedge}{ }^{\text {A }}-$ for SLATE or STATE, and for P2 items: ${ }^{-I_{-}}$ for PILE or PIPE).

The idea here was the possibility that in the critical letter context, participants might find the presence of the critical letter at least on some repetition trials sufficient reason to account for any recalled evidence of the repeated letter, and, hence, at least some reason not to report another one, resulting in a repetition deficit. The non-critical letter context, in contrast, does not match the critical letter on the repeated trials, and, hence, no reason not to recall the repetition letter from the cued position, producing no repetition deficit.

\section{Method}

Participants. Twenty-four students of both sexes participated in exchange for partial credit in an introductory or second-year psychology course.

Materials and Design. The materials and basic design were the same as described in Experiment 2: one-half of the trials requested full-report, the remainder partial-report, split here into two types of partial-report: critical-letter and noncritical letter contexts. Within each type of report, one-half of the trials were the RSVP of the same-length control item of the triplet, and the remainder were the RSVP of the repetition item. As in Experiments 1 and 2, participants were unaware of the required report task until after the RSVP of the word for that trial.

\section{Results and Discussion}

The data were analysed as in Experiments 1 and 2. Shown in Figure 3 are the mean proportion of correct responses to same-length control vs. repetition items, as a function of full-report, partial-report, non-critical letter context, and partial-report, critical letter context, for each of the P1 (e.g., SLATE vs. STATE) and P2 (e.g., PILE vs. PIPE) item-sets. Error-bars are the unique Fisher $\mathrm{LSD}_{.05}$ for each control vs. repetition comparison. As may be seen, and as in Experiments 1 and 2, full-report evinced large repetition deficits: control words were correctly reported significantly more frequently than were repetition words for both $\mathrm{P} 1(F(1,23)=$ $\left.55.90, M S E=0.0121, p<.0001, r_{p b}^{2}=0.71\right)$ and $\mathrm{P} 2$ $\left(F(1,23)=32.2000, M S E=0.0178, p<.0001, r_{p b}^{2}=0.58\right)$

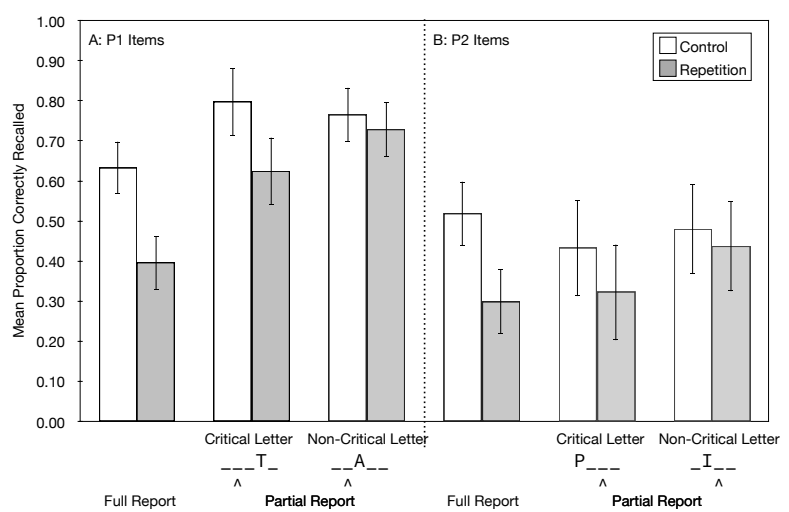

Figure 3. Mean proportion of correct recall in Experiment 3 as a function of repetition-type (control vs. repetition) for fulland partial-report (both critical vs. non-critical letter contexts) for both P1 (panel A:) and P2 (panel B:) items. The cue for the critical letter context included only the repeating letter in the nonspecified position with all other letter positions marked with an underscore and the cue for the non-critical letter context included only a non-repeating letter among the underscores. Error-bars are \pm Fisher $L S D_{.05}$ for the effect of repetition-type within each of the recall vs. partial-report (critical vs. non-critical letter contexts) conditions of the P1 vs. P2 item-sets.

item-sets. Similar results were found for the critical letter, partial-report context, although not as robustly as with full-report, for the P1 item-set $(F(1,23)=18.11, M S E=$ $\left.0.0196, p=.0003, r_{p b}^{2}=0.44\right)$, and at best marginally for the P2 item-set $(F(1,23)=3.62, M S E=0.0396, p=$ $\left..0695, r_{p b}^{2}=0.14\right)$.

However, as in the no-letter partial-report condition of Experiment 2, that was not the case for partial-report in the non-critical letter, partial-report condition. As can be seen in Figure 3, there is no evidence of a repetition deficit for either P1 or P2 item-sets: control words were not correctly reported significantly more frequently in the non-critical letter, partialreport condition than were repetition words in either of the $\mathrm{P} 1\left(F(1,23)=1.27, M S E=0.0126, p=.2713, r_{p b}^{2}=0.05\right)$ or the $\mathrm{P} 2\left(F(1,23)=0.59, M S E=0.0351, p=.4489, r_{p b}^{2}=\right.$ $0.03)$ item-sets.

Inspection of Figure 3 suggests an explanation. Comparing the two means of the control conditions and the two means of the repetition conditions of critical letter and non-critical letter partial-report for each of the P1 and P2 data-sets, we find that the principle effect is that the non-critical letter, partial-report condition increases retrieval of the repetition items relative to critical letter, partial report condition compared to that of the 
control items (which evinced no such effect) for items from both P1 (control: $F(1,23)=0.8519, M S E=0.0138, p=$ $.3656, r_{p b}^{2}=0.04$ and repetition: $F(1,23)=6.69, M S E=$ $0.0195, p<.0001, r_{p b}^{2}=0.23$ ) and P2 (control: $F(1,23)=$ $0.6954, M S E=0.0379, p=.4129, r_{p b}^{2}=0.03$ and repetition: $\left.F(1,23)=6.46, M S E=0.0244, p=.0183, r_{p b}^{2}=0.22\right)$. That is, relative to non-critical letter, partial-report, critical letter, partial-report appears to interfere with the report of repetition items, but not control items, presumably because the retrieval context of the repeated letter results in at least some reason not to report yet another one at the cued position.

\section{General Discussion}

From its initial demonstrations (Kanwisher, 1991; Kanwisher \& Potter, 1990; Park \& Kanwisher, 1994) to currently, repetition blindness is generally considered as an encoding/representational/perceptual phenomenon, revealing some fundamental facets of visual perceptual processing. But there have been a few exceptions, also almost from the beginning (notably in the work of Armstrong \& Mewhort, 1995; Fagot \& Pashler, 1995; Greene, 1991; Masson et al., 2000; Vokey \& Allen, 2002; Whittlesea et al., 1995; Whittlesea \& Podrouzek, 1995; Whittlesea \& Wai, 1997), arguing that rather than being wholly or mostly a perceptual phenomenon, such repetition deficits can profitably be seen as either in whole or in part phenomena of memory retrieval of the just presented event, reflecting instead some fundamental facets of short-term memory retrieval.

The most common dependent measure in repetition blindness tasks is that of full-report: participants are asked to report all elements (letters, words, colours, shapes, etc.) of a stimulus just presented in RSVP, with the resulting completeness of that report taken as a direct measure of that RSVP experience. Although some allowance typically is made for how that report may be attenuated relative to the actual encoding/representation/perception, it is assumed (presumably because of its immediacy) that any such attenuation is unbiased, not favouring one or another kind of the elements (such as a repeated letter) in the stimulus.

The three experiments reported here and the mechanisms we have suggested to explain the results, in contrast, were an attempt to challenge that assumption of unbiased report. In particular, because of the reconstructive, strategic, and heuristical nature of the retrieval-time processes required by these tasks (including full-report), full-report may in fact be biased against the report inter alia of repetitions following RSVP, providing a retrieval-time explanation for the phenomenon of repetition deficits without having to invoke any fundamental aspects of visual perceptual processing. The main results were these: if at report the context included the repeated letter as a retrieval cue (all letter partial-report and critical letter partial report) or would likely contain the repeated letter during report (full-report), repetition deficits were found, principally because report of the repetition letter, but not the control letter, was significantly reduced relative to the retrieval contexts not presenting the repetition letter (no letter partial-report and non-critical letter partial-report). Our explanation is that as long as the report context provides a reason to the participant to explain the retrieved evidence for the repeated letter, sometimes, but often enough to produce the repetition deficit, there is no need to report yet another. Thus, full-report (and all letter partial-report and critical letter partial report) are equivocal with respect to whether or not the participant actually encoded, represented, or perceived one or two instances of the repeated letter, and provide at best weak evidence that repetition blindness is an encoding/representational/perceptual phenomenon.

Do these results prove that it is retrieval-time rather than perceptual processes that are responsible for repetition deficits following RSVP? Of course not. It is easy to imagine that following the RSVP of a repetition item, the repetition of one of the elements strengthens the evidence that the repeated element occurred in the RSVP stream, but, as most theorists would have it, leaves no direct evidence that any repetition had occurred (think of the representation of each of the letters following the RSVP of STATE to be something analogous to $\mathrm{S}, \mathrm{T}, \mathrm{A}, \mathrm{E}$, in which the representation of the repeated $\mathrm{T}$ has a much larger presence than the other letters). Thus, the participant arrives at the report task with compelling reason to report that the repeated letter did occur during RSVP and, hence reports it in full-report along with as many other letters as can be retrieved, or reports it as the preferential response for no letter partial-report and non-critical letter partial-report as nothing in the perceptual record contradicts that it may have occurred at the cued position, thereby eliminating the repetition deficit, but, as the perceptual record shows, there is no reason in full-report (or all letter partial-report and critical letter partial report) to report it a second time, resulting in the usual repetition deficit. Unfortunately, for any kind of definitive resolution, except for the vague description of the perceptual (memorial?) record, this characterisation is as much a retrieval-time explanation as it is of something fundamentally pertceptual.

Our point is not to deny that there may be encoding/representational/perceptual aspects to repetition deficits but to point out that, to the the extent that there are, they are generally measured through a reporting process that responds to manipulations known to affect short term memory. Such influences are not surprising given that the reporting task is, in essence, a short term memory task. Retrieval-time effects on the reporting of a participants' experience should be considered in any interpretation of the rapid serial visual presentation of items. 


\section{References}

Armstrong, I. T., \& Mewhort, D. J. K. (1995). Repetition deficit in RSVP displays: Encoding failure or retrieval failure? Journal of Experimental Psychology: Human Perception and Performance, 21, 1044-1052.

Averbach, E., \& Coriell, A. (1961). Short-term memory in vision. Bell Systems Techinical Journal, 40, 309-328.

Averbach, E., \& Sperling, G. (1961). Short-term storage of information in vision. In C. Cherry (Ed.), Information theory (pp. 196-211). London: Butterworth.

Baylis, G. C., Driver, J., \& Rafal, R. D. (1993). Visual extinction and stimulus repetition. Journal of Cognitive Neuroscience, 5 , 453-466.

Epstein, R., \& Kanwisher, N. (1999). Repetition blindness for locations: Evidence for automatic spatial coding in an RSVP task. Journal of Experimental Psychology: Human Perception and Performance, 25, 1855-1866.

Fagot, C., \& Pashler, H. (1995). Repetition blindness: Perception or memory failure? Journal of Experimental Psychology: Human Perception and Performance, 21, 275-292.

Goldfarb, L., \& Treisman, A. (2011). Repetition blindness: The survival of the grouped. Psychonomic Bulletin $\mathcal{E}$ Review, 18(6), 1042-1049.

Greene, R. L. (1991). The Ranschburg effect: The role of guessing strategies. Memory $\mathcal{E}$ Cognition, 19, 323-317.

Harris, C. (2001). Are individual or consecutive letters the unit affected by repetition blindness? Journal of Experimental Psychology: Learning, Memory, and Cognition, 27, 761-774.

Harris, C., \& Morris, A. L. (2000). Orthographic repetition blindness. The Quarterly Journal of Experimental Psychology: Human Experimental Psychology, 53A, 1039-1060.

Hochhaus, L., \& Marohn, K. M. (1991). Factors in repetition blindness. Journal of Experimental Psychology: Human Perception and Performance, 17, 422-432.

Humphreys, G. W., Besner, D., \& Quinlan, P. T. (1988). Event perception and the word repetition effect. Journal of Experimental Psychology: General, 117, 51-67.

Kanwisher, N. (1987). Repetition blindness: Type recognition without token individuation. Cognition, 27, 117-143.

Kanwisher, N. (1991). Repetition blindness and illusory conjunctions: Errors in binding visual types with visual tokens. Journal of Experimental Psychology: Human Perception and Performance, $17,404-421$.
Kanwisher, N., Kim, J.-W., \& Wickens, T. D. (1996). Signal detection analyses of repetition blindness. Journal of Experimental Psychology: Human Perception and Performance, 22, 1249-1260.

Kanwisher, N., \& Potter, M. (1990). Repetition blindness: Levels of processing. Journal of Experimental Psychology: Human Perception and Performance, 16, 30-47.

Masson, M. E. J. (2004). When words collide: facilitation and interference in the report of repeated words from rapidly presented lists. Journal of Experimental Psychology: Learning, Memory, and Cognition, 30(6), 1279.

Masson, M. E. J., Caldwell, J. L., \& Whittlesea, B. W. A. (2000). When lust is lost: Orthographic similarity effects in the encoding and reconstruction of rapidly presented word lists. Journal of Experimental Psychology: Learning, Memory, and Cognition, 26, 1005-1022.

Park, J., \& Kanwisher, N. (1994). Determinants of repetition blindness. Journal of Experimental Psychology: Human Perception and Performance, 20, 500-519.

Sperling, G. (1960). The information available in brief visual presentations. Psychological Monographs, 74, 1-29.

Vokey, J., \& Allen, S. W. (2002). Repetition deficits, list context and word-class interactions in the RSVP of words in sentences. Canadian Journal of Experimental Psychology, 56, 98-111.

Whittlesea, B. W. A., Dorken, M. D., \& Podrouzek, K. W. (1995). Repeated events in rapid lists. Part 1: Encoding and representation. Journal of Experimental Psychology: Learning, Memory, and Cognition, 21, 1670-1688.

Whittlesea, B. W. A., \& Masson, M. E. J. (2005). Repetition blindness in rapid lists: Activation and inhibition versus construction and attribution. Journal of Experimental Psychology: Learning, Memory, and Cognition, 31(1), 54.

Whittlesea, B. W. A., \& Podrouzek, K. W. (1995). Repeated events in rapid lists. Part 2: Remembering repetitions. Journal of Experimental Psychology: Learning, Memory, and Cognition, 21, 1689-1697.

Whittlesea, B. W. A., \& Wai, K. H. (1997). Reverse "repetition blindness" and release from "repetition blindness": Constructive variations on the "repetition blindness" effect. Psychological Research, 60, 173-182. 